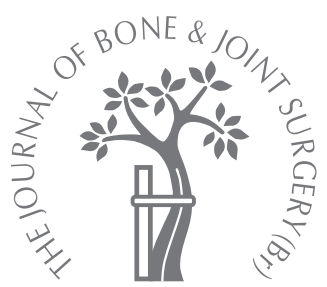
A. Alam,
K. Willett,
S. Ostlere
From The John
Radcliffe Hospital,
Oxford, England

\title{
The MRI diagnosis and management of incomplete intertrochanteric fractures of the femur
}

Incomplete intertrochanteric fractures do not extend across to the medial femoral cortex and are stable, without rotational deformity or shortening of the lower limb. The aim of our study was to establish whether they can be successfully managed conservatively. A total of 68 patients over a five-year period presented with a suspected fracture of the femoral neck and underwent an MRI scan for further assessment. From these, we retrospectively reviewed eight patients with normal plain radiographs but with an incomplete, intertrochanteric fracture on MRI scan. Five were managed conservatively and three operatively.

The mean length of hospital stay was 16 days for the conservatively-treated group and 15 days for those who underwent surgery; this was not statistically significant $(p>0.5)$ and all patients were mobilised on discharge. Although five patients were readmitted at a mean of 3.2 years after discharge, none had progressed to a complete fracture. We believe that patients with incomplete intertrochanteric fractures should be considered for conservative treatment.

Intertrochanteric fractures are extracapsular lesions which do not disrupt the blood supply to the femoral head and are not associated with nonunion or avascular necrosis. When displaced, their operative management involves a reduction of any anatomical deformity followed by internal fixation with devices such as the dynamic hip screw (DHS) (Synthes, Solothurn, Switzerland). However, if the calcar femorale is intact, the fracture is considered to be stable. ${ }^{1}$ Incomplete fractures have no deformity and do not need reduction. If they do not progress to complete fractures they do not necessarily require operative treatment, thereby avoiding significant peri-operative risks and complications.

\section{Patients and Methods}

We performed a search of the hospital reporting database system for all adult patients with suspected fractured hips following a fall, and who had undergone a subsequent MRI scan. We identified a total of 68 patients over a period of five years who had required an MRI scan either to diagnose or exclude a fracture. Two patients with greater trochanteric fractures identified on a radiograph also had an MRI scan to establish the full extent of the fracture line (Table I); 66 of the 68 patients had normal plain radiographs but clinical suspicion of a fracture.
Table I. MRI findings in the study group. The site of the fracture, if present, is indicated

\begin{tabular}{lc}
\hline Fracture location & Number of patients \\
\hline Incomplete intertrochanteric & 8 \\
Subcapital & 10 \\
Pubic rami & 9 \\
Greater trochanter & 2 \\
Acetabulum & 1 \\
Femoral head & 1 \\
Undiagnostic & 3 \\
No fracture demonstrated & 34
\end{tabular}

MRI was performed at a mean of 3.5 days (1 to 10) after the fall, on a $1.5 \mathrm{~T}$ magnet (GE Medical Systems, Wilwaukee, Wisconsin) in the coronal and axial planes using T1weighted and Short Tau Inversion Recovery sequences. We retrospectively reviewed the medical records and imaging of the eight patients with an incomplete intertrochanteric fracture and, within this subgroup, compared those patients managed conservatively with those managed operatively. Their length of stay in hospital was statistically compared using Student's $t$-test. Values for $\mathrm{p}<0.05$ were regarded as significant.

\section{Results}

A hip fracture was suspected in 18 patients despite normal plain radiographs; these patients were subsequently found to have a 


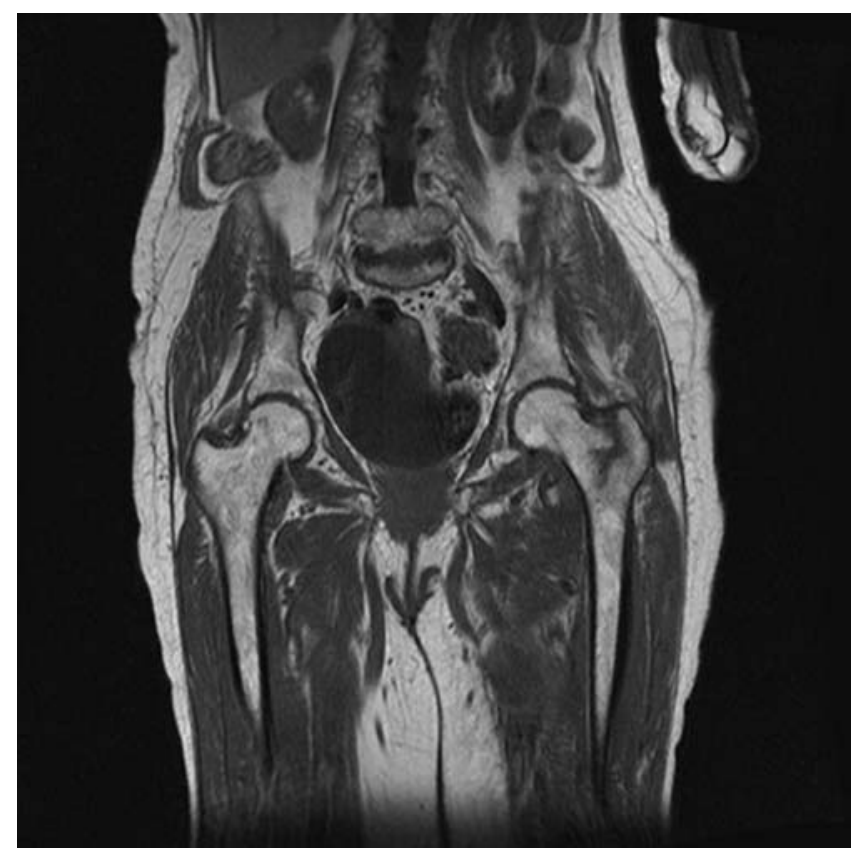

Fig. 1

A coronal T1-weighted MR image showing an incomplete intertrochanteric fracture of the left hip, which does not extend to the medial cortex. The plain radiograph did not show the fracture.

fracture of the femoral neck (ten intracapsular, eight intertrochanteric). The eight patients with an intertrochanteric fracture (five men, three women) did not have shortening of the lower limb, or an external rotation deformity on examination. Three patients, with a mean age of 61 years (SD 5.6) were managed with a DHS and five patients, with a mean age of 73.4 (SD 17.2) were considered to be a high anaesthetic risk and were, therefore, treated conservatively. The mean length of hospital stay in the conservativelytreated group was 16 days (SD 4.2) but 15 days (SD 3.0) for those treated with surgery; this was not statistically significant $(\mathrm{p}>0.5)$. All patients were mobilised with walking support on discharge. One patient developed a wound haematoma, which was the only known surgical complication in this group. No patient who had been treated conservatively was readmitted for a complete fracture although five were readmitted for unrelated reasons (two operative, three non-operative). The mean time of readmission after fracture was 3.2 years ( 2 to 5 ) although all five patients had been mobilising independently, with walking support, by the time of their readmission.

\section{Discussion}

It is known that patients with undisplaced fractures of the hip may have normal plain radiographs at the time of presentation. In such cases MRI is widely used to establish whether or not there is a fracture as the investigation is very sensitive to the haemorrhage and oedema which surrounds a fracture line while the linear nature of the lesion is char- acteristic. If there is no fracture of the hip, other lesions such as fractures of the pubic rami or soft-tissue injuries are often seen. MRI is a technique with high sensitivity, so the potential for misinterpreting and overtreating incidental findings is always present. This becomes less problematic as the role of MRI becomes better established in any particular clinical scenario. We have identified a specific pattern of abnormality seen in the intertrochanteric region in patients with a suspected fracture of the femoral neck. It comprises an intertrochanteric, linear signal abnormality originating at the greater trochanter and extending towards, but not reaching the medial cortex at the level of the lesser trochanter (Fig. 1). It may be misinterpreted as an occult, complete intertrochanteric fracture but is correctly interpreted as an incomplete fracture.

This impressive MRI abnormality in a patient who has a suspected fracture may convince the surgeon that internal fixation is required. In one study, the majority of patients with this lesion underwent internal fixation. ${ }^{2}$ From our study, we suggest that this lesion can only be diagnosed on MRI and that current evidence would suggest surgery is not indicated.

Although this is a retrospective study and post-operative imaging to assess union was not performed, no patient in the conservatively-managed group was readmitted for nonunion or progression to a complete fracture. We were also unable to determine retrospectively what proportion of patients had a suspected fracture but did not undergo an MRI scan. However, it has been the policy at our institution to perform an MRI scan on all patients with a suspected fracture of the femoral neck but with normal plain radiographs, so we believe that this proportion would be negligible.

It may also be argued that the functional demands of the conservatively-treated group were less than the operative group as the reason for not undergoing surgery was the high anaesthetic risk. Although this may have been the situation the five patients in the conservatively-treated group were mobilised on discharge while the three patients who were followed up at readmission were mobilising independently; this suggests a reasonable functional demand.

Others have found similar results with conservative management. Schultz et $\mathrm{al}^{2}$ arbitrarily suggested that only those incomplete intertrochanteric fractures which crossed the midsagittal line should be internally fixed. When they compared conservative with operative management, they found that the mean time from injury to walking was two days less in the surgical group but that there was no significant difference in the functional status between their two groups at clinical follow-up.

Before the introduction of MRI, many patients with hip pain following trauma, and normal plain radiographs, would have been treated conservatively. Some of these patients are known to have presented later with displacement of the femoral head, or avascular necrosis, because of a missed intracapsular fracture. However, we are unaware 
of any reports of missed intertrochanteric fractures, with normal radiographs on initial presentation, which have a subsequent fracture on a radiograph. This suggests that incomplete, undiagnosed intertrochanteric fractures are stable and likely to heal without complications. The role of MRI in patients with a suspected fracture of the femoral neck, but with normal plain radiography, should not be understated as it is particularly important to exclude an intracapsular fracture.

We have also observed this incomplete lesion as an intertrochanteric extension of greater trochanteric fractures. It has been suggested that MRI has a role in determining the extent of a fracture line in those patients who are known to have a greater trochanteric fracture. ${ }^{3-5}$ As with an incomplete intertrochanteric fracture, this is probably a common, previously unrecognised phenomenon, and should not automatically alter management.

In conclusion, incomplete intertrochanteric fractures are a relatively new MRI-specific diagnosis. As with any new finding on MRI, it is important not to overstate its signifi- cance, particularly as there is no historical evidence that these occult fractures progress. A trial of conservative management, avoiding the risks of surgery, should always be considered and the AO/ASIF classification ${ }^{6}$ may need to be modified to include incomplete intertrochanteric fractures.

No benefits in any form have been received or will be received from a commercial party related directly or indirectly to the subject of this article.

\section{References}

1. Evans EM. The treatment of trochanteric fractures of the femur. J Bone Joint Surg [Br] 1949;31-B:190-203

2. Schultz E, Miller TT, Boruchov SD, Schmell EB, Toledano B. Incomplete intertrochanteric fractures: imaging features and clinical management. Radiology 1999; 211:237-40.

3. Feldman F, Staron RB. MRl of seemingly isolated greater trochanteric fractures. Am J Roentgenol 2004;183:323-9.

4. Craig JG, Moed BR, Eyler WR, van Holsbeeck M. Fractures of the greater trochanter: intertrochanteric extension shown by MRI. Skeletal Radiol 2000;29:572-6.

5. Omura T, Takahashi M, Koide $\mathbf{Y}$, et al. Evaluation of isolated fractures of the greater trochanter with magnetic resonance imaging. Arch Orthop Trauma Surg 2000; 120:195-7.

6. Müller ME. The comprehensive classification of fractures of long bones. Berlin: Springer-Verlag, 1990 